first suggested.11 Furthermore, a thoracic duct fistula is difficult to keep draining for more than a few days, and once it becomes occluded bleeding is likely to recur.

In summary the control of oesophageal haemorrhage remains an unsolved problem, while the prognosis is determined largely by the patient's liver function. The desirability, timing, and nature of any of the possible surgical procedures are still not established.

1 Orloff, M. J., et al., Annals of Internal Medicine, 1967, 66, 165

2 Sherlock, S., British fournal of Surgery, 1964, 51, 746.

Hunt, A. H., St. Bartholomew's Hospital fournal, 1965, 69, Clinical Suppl. $11,13$.

Walker, R. M., Thorax, 1960, 15, 218.

Hunt, P. S., Johnston, G. W., and Rodgers, H. W., British fournal of Surgery, 1969, 56, 305.

' Mikkelsen, W. P., Review of Surgery, 1962, 19, 141.

Sobel, S., Kaplitt, M. J., Popowitz, L., Girardet, R. E., and Adamsons, R. J., Surgery, 1970, 68, 456.

Piccone, V. A., and LeVeen, H. L., Surgery, Gynecology and Obstetrics, $1967,125,66$

? Silva, Y. J., Surgery, Gynecology and Obstetrics, 1970, 131, 532

10 Dumont, A. E., and Mulholland, J. H., Annals of Surgery, 1962, 156, 668.

1 Kessler, R. E., Santoni, E., Tice, D. A., and Zimmon, D. S., Gastroenterology, 1969, 56, 538 .

\section{Prevention of Suicide}

Attempted suicide has long reached epidemic proportions, with an incidence of about 1 per 1,000 population per annum in our cities. It accounts for up to $15 \%$ of acute medical admissions, entails at least 50,000 psychiatric consultations per annum, and continues to increase at a rate of nearly $10 \%$ per annum. ${ }^{12}$ Viewed as attempts at self-destruction, many of these episodes appear to be half-hearted or histrionic, and the medical staff who have to deal with them sometimes feel a sense of irritation which they find difficult to conceal.

The acute risk to life often bears no relationship to the seriousness with which the attempt is made. A trivial overdose may be taken with more serious intent than a poisonous dose which nearly results in death. Any suicidal gesture carries a high risk of repetition and, owing to the inherent danger of the methods commonly adopted, a subsequent death rate of $1-2 \%$ per annum. ${ }^{34}$ It is still commonly held that people who talk about it never kill themselves, but several studies have shown that a high proportion of persons committing or attempting suicide give notice of their intent, and as many as half of them have recently consulted their doctor. ${ }^{45}$ Indeed, W. Ironside has put forward evidence to suggest that failure of the doctor to recognize and respond to the patient's needs may have been a factor in the precipitation of some suicides. ${ }^{6}$ 7

Suicide and attempted suicide are distinct but overlapping patterns of behaviour. Probably most people who commit suicide intend to die but some will survive and be labelled attempted suicide. But the great majority of those labelled attempted suicide are not addressing themselves to death: they are seeking, for a variety of motives, suspension of consciousness by methods so dangerous as sometimes to end in death. $\mathrm{N}$. Kessel ${ }^{8}$ has advocated the terms self-poisoning or self-injury for this form of behaviour, while N. Kreitman ${ }^{9}$ and colleagues preferred the description "parasuicide" to escape the constraints on thinking resulting from the implications of the term attempted suicide. Though the proportion of unintended deaths among people who attempt only selfpoisoning may remain fairly constant, they continue by their increase in number to add to the total of people killing themselves and so further blurr the distinction between suicide and self-injury.
Early recognition and prompt treatment of serious psychiatric disorder, particularly depression and alcoholism, are of prime importance in the prevention of suicide. In both suicide and self-poisoning the act is often impulsive and dependent on the ready availability of a suitable poison. Difficulty in obtaining large quantities of drugs on prescription or over the counter might deter many victims. The contribution of formal psychiatric disorder to self-poisoning or parasuicide is more difficult to estimate. The act is often an incident of crisis in a psychological disturbance or social predicament rather than the symptom of a disease treatable by pharmacological means. Faced with a situation calling for skills in which he may lack training, the doctor is often tempted to refer such patients to psychiatric clinics, frequently with a delay which ensures that the crisis has resolved (perhaps inappropriately) by the time help is offered. Crisis intervention techniques $^{10}$ may be more suitable in these circumstances than more orthodox forms of treatment and may account for the apparent success of emergency counselling services such as the Samaritans. ${ }^{11}$ In a study of deliberate self-poisoning or self-injury presenting to the casualty department of a London teaching hospital S. Greer and C. Bagley ${ }^{12}$ reported that despite injunctions from the Ministry of Health ${ }^{13} 22 \%$ of the patients presenting were not referred for psychiatric opinion; for a further $36 \%$ psychiatric contact was limited to one or two interviews; and only $42 \%$ received prolonged treatment. In these circumstances it is encouraging to find that the patients offered treatment were less likely to make a further attempt or actually to commit suicide than the others, while the group not seen by a psychiatrist carried the highest morbidity. Thus it does appear that help offered at the time can bring real benefit.

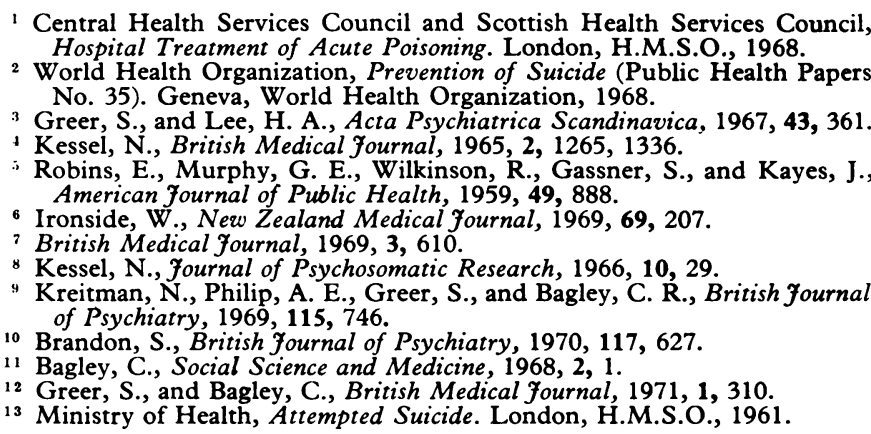

\section{Dull in Tooth and Mind}

Defects in teeth, particularly crown defects, are more common among mentally retarded children. ${ }^{1}$ Both the teeth and the nervous system are derived from the embryonic ectoderm. In a recent survey $H$. J. Cohen and $H$. Diner ${ }^{2}$ investigated the significance of developmental dental enamel defects in three groups of children: 215 suspected of a neurological or mental disorder, 139 children of a similar low-income background attending a day centre, and 150 from middle class backgrounds attending nurseries or kindergartens. All three groups were comparable in age, 2-14 years, and all had known dental data. Developmental enamel defects were most common in group 1, and particularly in those with neurological deficit. Enamel defects were least common in the children in group 3. Cohen and Diner also noted a definite correlation between enamel defects and low intelligence quotient, where neurological defects, unspecified, were also noted. 
The dental enamel defects may be in the nature of hypoplasia, hypocalcification, or hypomaturation, and are considered to be due to antenatal, and perhaps unreported, disturbances. The requirements for normal dental development are not known, but nutrition must play a part, and it can be altered by disease processes. The ectodermal origin of both enamel and the nervous system, with the suggestion that defects in enamel are seen when there is neurological deficit and also a low intelligence lends support to the possibility that the insult occurred early in pregnancy. This is of importance in the elucidation of the aetiology of some neurological and mental defects. It is also a useful pointer for the dental practitioner and paediatrician for a closer examination of the child's intellectual ability and neurological status.

Kraus, B. S., Clark, G. R., and Oka, S. W., American fournal of Mental Deficiency, 1968, 72, 905 .

Cohen, H. J., and Diner, H., Pediatrics, 1970, 46, 737.

\section{Tropical Splenomegaly Syndrome}

Considerable progress has been made in recent years in the definition and understanding of the tropical splenomegaly syndrome, ${ }^{1}$ and work published in this issue of the B.M.F. confirms that effective drug treatment is available. This syndrome may soon join those many disorders for which successful treatment has been found before the full story of the pathogenesis has been unravelled.

Malarial infection is highly associated with this condition, and it has thus been considered reasonable to give patients antimalarial therapy. Indeed, proguanil has been used for many years in Nigeria in a daily dose of $100 \mathrm{mg}$, with an excellent response. ${ }^{2}$ Working in Uganda, Dr. P. C. Stuiver and his colleagues have now completed a randomized, doubleblind trial of malaria prophylaxis in patients with the tropical splenomegaly syndrome, all of whom had hepatic sinusoidal lymphocytosis. Their report is at page 426. After two weeks on $15 \mathrm{mg}$ primaquine daily the treated patients received chloroquine phosphate $300 \mathrm{mg}$ base weekly. All 14 patients on this treatment showed a reduction in estimated spleen volume, and in 11 patients this was by more than $50 \%$ of the original spleen volume. In the 15 controls on placebo no patients' spleen volume was reduced by more than $50 \%$.

The younger patients tended to have larger spleens initially and also responded better to treatment than the older ones. Relief of symptoms such as chronic abdominal discomfort, fevers, and weakness was greater in the treatment group, and their haemoglobin levels also improved. After 12 weeks the size of the spleen was still falling in most of the treated patients, and it seemed likely that treatment would have to be continued.

The Ugandan study required that the patients return to the hospital clinic once a month for periods up to 12 months or more. This is obviously not always possible in tropical and underdeveloped countries, and it is also difficult to ensure, in the tropics or elsewhere, that drugs are regularly taken over a long period of time. For this reason, a study has been carried out in Zambia, and is reported at page 429 by Dr. M. N. Lowenthal and his colleagues, with a depot injectable antimalarial, cycloguanil pamoate, either alone or combined with proguanil. Despite considerable variation in dosage and in the duration and frequency of treatment, there was good evidence to show that this form of therapy can lead to reduction in spleen size.

Intramuscular cycloguanil pamoate alone was given to 12 patients, and eight of them showed a significant reduction in spleen size, as did two out of three patients treated with cycloguanil and proguanil. Repeat liver biopsy was performed in five who responded to treatment, and in four of these there was a definite reduction in hepatic sinusoidal lymphocytosis.

The Nigerian study by A. S. Sagoe ${ }^{3}$ indicated a clear-cut distinction before treatment between those who responded to treatment and those who did not. The responders showed very high pre-treatment levels of $\operatorname{IgM}$, and their blood lymphocytes responded normally to phytohaemagglutinin. In the Zambian study the IgM findings did not agree completely with those of the Nigerian study, and it is clear that further investigation of these changes is needed.

The two papers this week confirm the clinical effectiveness of prolonged malarial prophylaxis in the treatment of the tropical splenomegaly syndrome, as previously reported from West Africa. The optimal duration of antimalarial administration required is not yet established, and even after long periods of apparently successful treatment patients may relapse on discontinuing their antimalarial drugs. It may be that these particular individuals will require treatment as long as they are at risk from malarial infection.

While there seems little doubt that malaria is the fundamental cause of the tropical splenomegaly syndrome, only a very small proportion of people exposed to malaria develop gross splenomegaly. It has thus been postulated that the condition is due to an abnormal host response to malaria, or to malaria acting in association with one or more additional factors, or to some specific variant of the malarial parasite. In Uganda there is some evidence that Plasmodium malariae is commoner in patients than controls, but this has not been confirmed elsewhere. It seems likely from the results of antimalarial therapy described in these papers that the malaria parasite itself may be providing a continuing antigenic stimulus, which results in a benign lymphoproliferative disorder affecting predominantly the spleen and liver. The elimination of this hypothetical low-grade malarial parasitaemia would be a simple explanation for the effectiveness of therapy.

The question of malarial immunity becomes more complex as more information becomes available, and a recent symposium on the role of serum antibodies in malarial immunity stresses the need to recognize malaria as a human disease and not merely as an interesting immunological phenomenon. ${ }^{+}$ The wider aspects of immunity to malaria have recently been described in detail ${ }^{5}$ and the problems of the sero-epidemiology of malaria have also been critically reviewed. ${ }^{6}$ Malaria and its associated disorders remain among the most important problems of the tropical countries and must remain high on the list of priorities for research investment there.

British Medical fournal, 1970, 3, 360

Watson-Williams, E. J., and Allan, N. C., British Medical Fournal, 1968, 4, 793 .

Vole, A. S., British Medical fournal, 1970, 3, 378. Tropical Medicine and (ther, A., Transactions of the

Hygiene, 1971, 65, in press.

Bruce-Chwatt, L. J., fournal of Parasitology, 1970, 56, No. 4, Section 2, p. 552 . 